\title{
EL CUERPO COMO BASE DE LA ORGANIZACIÓN COGNOSCITIVA Epistemología genética y fenomenología*
}

\section{Ma. Elena Candioti de De Zan}

"Si la verdad es una organización de lo real, la cuestión previa es comprender cómo se organiza una organización, y ésta es una cuestión biológica...Pero lo propio de la vida es superarse sin cesar, y si buscamos el secreto de la organización racional en la organización vital, sin exceptuar sus superaciones, el método consiste en tratar de comprender el conocimiento por su construcción misma, lo que nada tiene de absurdo, puesto que es esencialmente construcción". 1

Cuando planteamos las relaciones entre el conocimiento y la corporalidad, parece inevitable el paso por los extensos estudios piagetianos acerca de la conformación del conocimiento a partir de las acciones corporales, en un proceso de desarrollo que si bien no se reduce a lo biológico, encuentra allí sus raíces. Queremos sin embargo discutir algunas de sus conclusiones y señalar

*Este trabajo fue presentado en las Jornadas de Filosofía de la Universidad Nacional de Cuyo, Mendoza, junio de 1996.

${ }^{1}$ Piaget, J., Biología y conocimiento, Méjico, Siglo XXI, 1985, p. 334 
algunas deficiencias que advertimos a la luz de los análisis fenomenológicos acerca de la teleología de la experiencia, su intersubjetividad e historicidad.

Tanto la epistemología genética como la fenomenología se enfrentan al "objetivismo", entendido como aquella mirada unilateral que desconoce las funciones activas del sujeto, priorizando el "dato". Oponiéndose críticamente a los supuestos que condicionaron al empirismo y al racionalismo modernos, como así también al positivismo y a las interpretaciones racionalistas o encubiertamente positivistas de la filosofía clásica, ambas afirman la idea de un sujeto constructivo, procurando mostrar las falencias de las concepciones mecanicistas del cuerpo y de los dualismos a las que se vieron frecuentemente asociadas. En ambos casos se abandona la idea del cuerpo como objeto, para concederle una activa participación en las funciones cognoscitivas a través de un proceso constructivo orientado temporalmente. Sin embargo hay importantes diferencias en cuanto se trata de precisar ciertas cuestiones centrales, como la relación del sujeto con el mundo circundante, el modelo de conocimiento (y en última instancia de racionalidad) que consideran, y el modo en que articulan contingencia y universalidad.

La convicción básica de que puede encontrar en la Biología la base adecuada de explicación, conduce a Piaget a plantear el problema del conocimiento desde una perspectiva específica, adoptando ciertos marcos conceptuales que permiten teorizaciones unificadoras, susceptibles de contrastación experimental. "Los procesos cognoscitivos se nos manifiestan, entonces, simultáneamente como la resultante de la autorregulación orgánica, cuyos mecanismos esenciales reflejan, y como los órganos más diferenciados de esta regulación en el seno de la interacciones con el exterior, de manera que terminan, con el hombre por extender éstas al uni- 
verso entero" 2 . El conocimiento, entendido a partir de la acción, supone esquemas de asimilación y acomodación progresivos. Conocer no es copiar lo real, sino transformarlo a fin de comprenderlo en función de los sistemas de transformación a los que están ligadas las acciones. ${ }^{3}$ Ahora bien, estos esquemas traen una organización que nos vincula a lo biológico. Si bien la mayoría de los esquemas, no se deben a un montaje hereditario ya dado, sino que se construyen gradualmente y se modifican por acomodación, no son independientes de factores internos; un esquema no tiene nunca un comienzo absoluto, sino que se deriva por diferenciaciones sucesivas de esquemas anteriores que se van remontando hasta las bases organizativas propias de la especie. Los esquemas cognoscitivos se derivan uno de los otros, y en última instancia dependen de coordinaciones orgánicas, de tal modo que el proceso en su conjunto responde a procesos de autorregulación, construyendo para ello las estructuras apropiadas. ${ }^{4}$

El pensamiento es de algún modo "biologizado", en cuanto sus estructuras son concebidas a partir del desarrollo de un factor vital endógeno, en interacción con el medio. ${ }^{5}$ Hay una cierta

${ }^{2}$ Piaget, J., Biología y Conocimiento, op.cit. p. 26

3"Decir que todo conocimiento supone una asimilación, y que ella consiste en conferir significaciones, ...es tanto como afirmar que conocer un objeto implica su incorporación a esquemas de acción, y esto es verdad desde las conductas sensorio-motrices elementales hasta las operaciones lógico matemáticas superiores." Piaget, J., Biología y conocimiento, op.cit. p. 9

${ }^{4}$ Ibid., p. 75-76

${ }^{5}$ En esta comparación de los mecanismos cognoscitivos con los procesos orgánicos, Piaget procura evitar tanto el vitalismo que quiere encontrar inteligencia en todo, como el reduccionismo que asimila fácilmente las funciones superiores del conocimiento a comportamientos elementales. Piaget, J., Biología y conocimiento, op.cit., p. 1 
direccionalidad cognoscitiva que define el proceso desde las etapas sensorio-motrices hasta la formación de las estructuras abstractas lógico-matemáticas ${ }^{6}$, a través de procesos autorregulativos.

Piaget busca mediante un principio dinámico, dar cuenta de esta "racionalización" de la experiencia lograda en cuanto ésta es susceptible de ser organizada por las operaciones del pensamiento, que en su reversibilidad logran superar un curso de experiencia irreversible y caótico. Es por medio de este proceso epigenético que se desarrolla lo que podría considerarse una "embriología de la razón", en cuanto explicita el desarrollo de los procesos cognoscitivos, hasta la consecución de ciertas formas (las estructuras lógico-matemáticas) consideradas como la meta necesaria.

El dinamismo adaptativo que regula la relación entre individuo y medio circundante, se orienta a lo que se consideran las

${ }^{6}$ Para comprender esto es necesario -señala Piaget- evitar simplificaciones. "Convendrá distinguir entre las conductas cognoscitivas adquiridas, y sobre todo entre las formas superiores de la inteligencia, dos aspectos esencialmente diferentes en lo que respecta al papel que desempeñan en su elaboración las actividades del sujeto o del organismo, por un lado, y los objetos mismos o el medio, por otro: éstos son los aspectos lógico-matemáticos y los aspectos exógenos (aprendizaje empírico y conductas experimentales) del conocimiento. Ahora bien, las estructuras lógico-matemáticas que, evidentemente suponen una parte preponderante de actividad y de organización internas...se manifiestan ya (aunque mezcladas constantemente con los datos exteriores, de los que se disociarán solamente en las etapas superiores del pensamiento) en todos los niveles de las conductas adquiridas, e inclusive de la percepción, por no decir también en ciertos instintos: las "Gestalt" perceptivas traen consigo una geometrización...”. Piaget, J., Biología y conocimiento, op.cit., p. 5. Sobre los aspectos figurativos y formales del conocimiento: Vuyck, R., Panorámica y crítica de la epistemología genética de Piaget 1965-1980, Madrid, Alianza, 1984, Tomo I, op.cit., p. 119 a 121. 
estructuras más elevadas por sucesivos momentos que responden a lo que puede considerarse una ortogénesis. El principio dinámico del desarrollo es interpretado a partir de los conceptos de reversibilidad y equilibración $n^{7}$, dando cuenta así del sentido racional de la ontogénesis cognoscitiva. La intemporalidad de la reversibilidad y el equilibrio son los puntos finales de un proceso irreversible de construcción de las configuraciones cognoscitivas, en cuanto indican que se ha logrado la necesaria descentración de la experiencia. La "equilibración", considerada como el motor de los procesos, es entendida de manera reductiva, limitada a cumplir la función de posibilitar una nueva forma de equilibrio conducente a las etapas superiores del desarrollo. Asimilación y acomodación, aún cuando se entiendan con flexibilidad, son funciones insertas en un proceso de algún modo predeterminado por esta ortogénesis inmanente que culmina en las estructuras propias del pensamiento adulto. De esta forma se privilegia una única forma de acceso, quedando como un residuo no racionalizable toda otra forma de pensamiento que no se inserte en los marcos lógicos del pensamiento matemático o físico-matemático. La preocupación por explicar la construcción de las estructuras formales que posibilitan el conocimiento, desplaza al tema de la constitución del sentido, y más aún, de la configuración de múltiples marcos significativos diferentes de este tipo de racionalidad.

De acuerdo a esto, tendríamos que admitir la existencia de ciertos supuestos "cientificistas" en la epistemología de Piaget, concibiendo todo el proceso cognoscitivo desde esta óptica que

\footnotetext{
7Sobre la idea de equilibración: Vuyck, R., op.cit., p. 36
} 
privilegia el conocimiento físico-matemático ${ }^{8}$, en el logro de estructuras generalizables.

Pero podemos objetar a Piaget que la relación entre el sujeto corporal y el mundo no sigue necesariamente las reglas de un pensamiento formal o la de una experiencia matematizada. $\mathrm{Si}$ por mundo entendemos el mundo de la cultura (Lebenswelt) referente desde el cual se comprende toda consideración objetivay lo consideramos en toda su fluidez, complejidad y variabilidad, advertimos entonces la estrechez de los análisis piagetianos al circunscribir a una determinada forma de estructuración las posibilidades del pensamiento. La perspectiva del orden matematizante es sólo una de las tantas posible, y es por esto discutible que la relación con el mundo tienda a ajustarse a funciones invariantes, y que las metas cognoscitivas puedan explicarse desde un modelo que toma como pauta la estructura de la racionalidad científica.

Ahora bien, no sólo el modelo de conocimiento que se presenta como la etapa madura del pensamiento humano está restringido, sino que el tratamiento mismo que se hace de estas cuestiones queda determinado por los marcos teóricos y los procedimientos propios de la ciencia empírica.

Tal como la fenomenología insistentemente ha mostrado, la genealogía de la experiencia no puede entenderse deductivamente a partir de conceptos acerca de la naturaleza, elaborados con la metodología y criterios propios de estas ciencias,

${ }^{8} \mathrm{El}$ creciente interés por el pensamiento científico se pone de manifiesto en Piaget, J., Recherches sur la généralisation. 1978. Este interés se traduce también en los estudios acerca de los mecanismo comunes del pensamiento infantil y el pensamiento científico. Piaget, J. y García, R., Psicogénesis e historia de la ciencia, Méjico, Siglo XXI, 1992. - Cf. Vuyck, op.cit., p. 37 
sino a partir de las estructuras de este campo significativo preobjetivo. Por esto, más que la transposición de categorías científicas que muestran el proceso cognoscitivo como una prolongación de la constitución biológica, se trata de explorar -siguiendo la consigna fenomenológica- este ámbito originario de constitución de sentido, previo a toda reducción o abstracción operada por los procedimientos que constituyen los diferentes campos disciplinarios.

Así considerada, la relación del sujeto con el mundo es más compleja de lo que puede explicarse desde procesos biológicos de equilibración, y el cuerpo no es sólo un organismo regulado por funciones adaptativas, sino sujeto corporalmente situado, y en reciprocidad con otros. Si el cuerpo es entendido como "Cuerpo propio" (Leib), más que a una legalidad natural responde a un movimiento total de la existencia humana; la dinámica propia del complejo biológico se articula con la autonomía del proyecto humano.

El cuerpo es el sujeto mismo que se prolonga asumiendo una naturaleza. Esto significa que es algo más que su constitutivo material. La inserción en el mundo espacio-temporal requiere el "cuerpo propio" como ese sistema de órganos que se mueve egológicamente en su operar, y por el cual interviene en el mundo circundante, siendo consciente de él en la forma del "yo puedo", "yo hago", etc. ${ }^{9}$

El cuerpo abre las perspectivas y organiza motrizmente el horizonte; es base de la organización de la experiencia en cuanto estructura un marco espacial y temporal. En los escritos

${ }^{9}$ Husserl, E., La crisis de las ciencias europeas y la fenomenología trascendental, Méjico, Folios,-1984, p. 110 
husserlianos se encuentran afirmaciones acerca de una organización de la experiencia a nivel corporal, sin que esto implique afirmar que la naturaleza corporal -y por su intermedio la subjetividad-, esté regida por una causalidad en tercera persona. Para precisar esto debemos investigar los procesos perceptivos de la cosa material, donde evidentemente los órganos corporales desempeñan una función primordial. El perspectivismo de la experiencia está dado por su relación al órgano sensorial y a las capacidades corporales; la relatividad de los fenómenos está determinada por la intencionalidad orgánica que, por supuesto, es limitada. Pero también el cuerpo en su operatividad cinestésica, es el que permite la variación de la experiencia, la reiteración desde diversos ángulos. El significado del mundo se construye a partir de esta corporeidad operante.

El yo concreto en cuanto corporal es así un "yo me muevo", un "yo hago" que va configurando intencionalmente el mundo. Los movimientos cinestésicos se vinculan entre ellos en orden a un fin que los motiva. Las diversas presentaciones surgen también intencionalmente: "Si me muevo así, entonces veré tal aspecto" (Wenn-so). Las síntesis que se realizan a nivel sensible implican por tanto una espontaneidad organizativa; no se trata (como en Kant) de una sinopsis que estructura receptivamente un dato; la aparición del dato exige la actividad del sujeto, y esta actividad es la que da razón de su manifestación. El curso de las diversas manifestaciones que se integran sintéticamente está dirigido espontáneamente, en una libertad de experiencia que a la vez que define un horizonte teleológico ( temporal), define también un horizonte espacial ${ }^{10}$.

${ }^{10}$ Landgrebe, L., Fenomenología e historia, Venezuela, Monte Avila Ed., 1975 p. 164 
La síntesis de pasividad y actividad se produce desde el ámbito perceptivo: a la pasividad de la recepción hylética se conecta necesariamente la capacidad de disponer de una motricidad que me presenta al mundo de manera originaria, antes de toda forma de pensamiento lógico. En esta síntesis se revela simultáneamente mi ser corporal como posibilidad práctica y la realidad como aquello sobre lo cual puedo ejercer mi actividad. "Las cosas están entretejidas con las habitualidades y las pasividades del yo, con la historia originaria, corpórea, del yo mismo. En un sentido nuevo, el cuerpo, así entendido, es un órgano"11. Es también conciencia de mi relación con el mundo en cuanto orientado a fines y motivos, y no determinados por fuerzas exteriores. El "yo puedo" está conectado al "yo quiero", a un movimiento dirigido, lo cual significa que las cinestesis y sus aspectos noemáticos correlativos tienen un orden, una organización que surge de una motivación.

La unificación se opera espontánea y teleológicamente, a partir de una operatividad corporal organizativa que implica a la vez la constitución del horizonte espacial de la experiencia y de su temporalidad. El cuerpo es el aquí absoluto desde donde se despliega el mundo, y el que "activiza" las sensaciones según cierta direccionalidad. Es así lo que constituye el campo primordial donde toda experiencia aparece, a la vez que la torna posible; en este sentido es un "apriori" (condición necesaria y posibilitante). Es el "punto cero" -afirma Husserl, expresión que acentúa posteriormente Merlau Ponty en cuanto lo considera el "pivote" del mundo-.

Son innumerables los pasajes en que Merlau Ponty insiste en esta función constitutiva de la corporalidad. El cuerpo es lo

\footnotetext{
${ }^{11}$ Paci, E., Función de las ciencias y significado del hombre", Méjico, Fondo de Cultura Económica, 1968, p. 42
} 
que mantiene con vida el espectáculo visible, lo anima y lo alimenta interiormente, formando un sistema con él; no sólo abre perspectivas sino que realiza además el nucleamiento, el nexo que hace posible la unidad objetiva. El "esquematismo corporal" es la clave de esta operatividad: permite aceptar el cuerpo como un todo, a la vez que posibilita " la unidad de los sentidos y la unidad del objeto" ". Es el cuerpo el que realiza la síntesis perceptiva en una síntesis pre-lógica ${ }^{13}$, el que "anuda" los momentos del tiempo ${ }^{14} \mathrm{y}$ la diversidad de los datos; es la "textura común" de todos los objetos, el instrumento general de mi comprensión ${ }^{15}$. La unidad preobjetiva de la cosa es así el correlato de la unidad pre-objetiva del cuerpo.

\section{La corporalidad aparece siempre como condición} posibilitante de la experiencia. Pero hay algo más: es también modalizante de ella; los diferentes modos de aparición están dados por diferentes modos de relacionarse al cuerpo, de tal modo que éste realiza una especie de "montaje" del mundo ${ }^{16}$.

\footnotetext{
${ }^{12}$ Merlau-Ponty, M., Fenomenología de la percepción, Barcelona, Ed. Península, 1975 , p. 247

${ }^{13}$ Ibid., p. 248

${ }^{14}$ Ibid., p. 254

${ }^{15}$ Ibid., p. 250. Cf. p. 362-5

16“...Mi experiencia desemboca en las cosas y se trasciende en ellas, porque se efectúa siempre en el marco de cierto montaje respecto del mundo, montaje que es la definición de mi cuerpo. Las magnitudes y las formas solamente modalizan esta presa global sobre el mundo. La cosa es grande si mi mirada no puede envolverla; es, por el contrario pequeña, si la envuelve de sobra, y las magnitudes medias se distinguen una de otra según que, a distancia igual, dilaten más o menos mi mirada o que la dilaten igualmente a distancias diferentes. El objeto es circular si, igualmente próximo de mí por todos sus lados, no impone al movimiento de mi mirada ningún cambio de curvatura, wo si los que le impone son imputables a la presentación oblicua, según la ciencia del mundo que se me da con mi cuerpo". Merlau-Ponty, Maurice, Fenomenología de la percepción, op.cit., p. 317
} 
Podría objetarse desde una óptica piagetiana que también en la epistemología genética las acciones corporales son las que permiten la estructuración espacial y temporal ${ }^{17}$; pero un examen más atento nos revelará las profundas diferencias de estas nociones de espacio y tiempo con la idea de "horizonte" significativo que, desplegando su espesor histórico, co-determina el sentido de la experiencia.

En cuanto sujeto, el cuerpo no se ajusta a una causalidad material ${ }^{18}$ sino que su modo de comportamiento es el que corresponde al hecho de ser el cuerpo de un yo personal. El cuerpo posee ya un estrato perteneciente a lo anímico que no es referido a él por nosotros mediante una consideración alusiva, sino que está allí de antemano, y por tanto intuitivamente, como estrato

17“"Pero es claro que si nuestros ojos fuesen distintos...o si no los tuviésemos, y si no estuviésemos dotados de manipulación ni de locomoción, con un sistema nervioso tan perfeccionado y cerebralizado, nuestro universo cognoscitivo sería muy diferente. Y sobre todo, si viviéramos con órganos análogos, pero a escala totalmente diferente, como la del átomo, nuestros conceptos fundamentales quedarían trastornados, y no sólo a causa del espectáculo, sino también de los medios de acción". Piaget, J., Biología y Conocimiento, op.cit., p. 249

${ }^{18}$ Esta mediación de lo corporal nos pone en la difícil situación de tener que considerar a la vez su función constitutiva y su naturaleza material. El modo de participación del sujeto en la naturaleza tal vez resulte algo difuso en la filosofía de Husserl. El cuerpo se manifiesta como un "oscuro transfundo" de la subjetividad personal, que marca su dependencia de la sensibilidad, y por lo tanto, de lo material Cf. Husserl, E., Ideas III, Husserliana, Tomo V, p.118. En su ser espiritual el yo reposa sobre un "trasfondo de vivencia, y un trasfondo de naturaleza (mi naturaleza) que se anuncia en la agitación de las vivencias. Cf.Husserl, E., Ideas II, Husserliana IV, p. 161. El cuerpo es el aspecto "natural" del yo, aunque lo natural debe entenderse en este caso de un modo especial, sin identificarlo con los procesos causales. 
aperceptivo correspondiente a la totalidad del cuerpo mismo. Esta dimensión es lo que rescata a la subjetividad de su anonimia y la inscribe en una operatividad más compleja y profunda que es la de la vida espiritual operante.

No hay legalidad biológica que determine por sí sola el curso de la conciencia; si el cuerpo es "condición", sólo lo es en cuanto hace posible la temporalidad constitutiva, abriendo un horizonte teleológico.

En este sentido es también soporte y centro de la intersubjetividad; cada perspectiva particular se correlaciona con una totalidad, se conecta a otras perspectivas posibles insertándose en un proceso definido por un horizonte abierto al infinito. El horizonte total no se agota en mis propias representaciones, trasciende las propias perspectivas y remite a otras, y en este sentido, la corporalidad no sólo es lo que define un centro, sino también lo que hace posible la integración ${ }^{19}$. El cuerpo es el medio comunicativo que hace posible convertir a una pluralidad de sujetos en comunidad intersubjetiva; es lo que permite a nuestra realidad ser una realidad social. Es así el medio necesario de toda operación, el conjunto de condiciones concretas por las cuales puede actualizarse la función constitutiva.

Mirado desde esta óptica, y teniendo en cuenta estas múltiples funciones corporales, el sujeto epistémico que muestra Piaget se advierte como una imagen esquematizada y empobrecida, una abstracción, y no el sujeto real y viviente en el sentido pleno de la palabra. Si bien se insiste en la autonomía y creatividad, este sujeto debe transitar por etapas sucesivas de construcción de estructuras posibilitantes que son comunes (generalizables a todos los su-

${ }^{19}$ Paci, E., op.cit., p. 142 
jetos) y secuencias que son inevitables.

La comprensión piagetiana del proceso de ontogénesis muestra una direccionalidad, pero esta se agota en el desarrollo diacrónico de estadios concebidos como un progreso dialéctico de equilibración en orden a ciertas metas prefijadas ${ }^{20}$. La epistemología piagetiana y su fundamentación en una psicología objetivista esconde, tras el uso del método empírico, el resurgimiento de una absolutización del pensamiento racional que, tras su apariencia de objetividad, reitera en perspectiva genética una "Religión de la Razón" - (Religion der Vernunft) ${ }^{21}$.

Esta transposición de categorías biológicas al orden de la experiencia humana nos hace preguntar hasta qué punto ha superado Piaget aquel tratamiento de la corporalidad que le impone una legalidad en "tercera persona".

Más que de una teleología de la experiencia humana, la perspectiva biologizante conduce a admitir una "teleonomía", es decir, una determinación causal en perspectiva cibernética ${ }^{22}$.

${ }^{20}$ Piaget, J., Biología y conocimiento, op.cit., p. 336

${ }^{21}$ Liebsch, Burkhard. "Spuren einer anderen Natur. Piaget, Merlau-Ponty und die ontogenetische Prozesse". (Übergänge, Bd. 24). München 1992. W. Fink, 434 S., en Herzog, M., "Entwicklungsdenken bei Piaget und Merlau-Ponty". Philosophische Rundschau. Band 41- Heft 4. Dezember 1994.

${ }^{22}$ Vuyck, R., op.cit., p. 59.- Cf. Boden, M., Piaget. Madrid, Cátedra, 1982. Cap. 7. Cf. Piaget, J., Biología y conocimiento, op.cit., p. 334. "Tratándose, pues, de elegir entre el innatismo y la construcción, y dando por sentado que el espíritu humano es un producto de la organización biológica antes que predomine en él la cultura colectiva, los hechos parecen indicar que el mecanismo orgánico que desempeña el papel fundamental en esta génesis no es la herencia, sino un sistema de autorregulaciones. Considerar las estructuras matemáticas como innatas presenta, en efecto, los más graves problemas biológicos 
De este modo, el espacio concedido a la historicidad del conocimiento humano, en cuanto ésta define un horizonte semántico es mínimo y elemental ${ }^{23}$. El ejercicio del método histórico-crítico pareciera eximir a Piaget de este reproche. Sin embargo, el recurso a la historia para establecer la significación epistémica requiere "formular el problema en término de "vecciones", es decir de evolución de las normas de una escala que permite discernir etapas". La intención de establecer convergencias y parentescos entre los estudios histórico críticos y psicogenéticos, responde a la razón profunda de "reencontrar en todos los niveles instrumentos y mecanismos similares, no sólo en las interacciones elementales entre sujetos y objetos, sino muy particularmente en la forma en que un nivel condiciona la formación del siguiente". Si bien encontramos en Piaget algunas páginas que suponen una mayor consideración de la historia y del rol de la transmisión cultural en los procesos cognoscitivos ${ }^{24}$, los análisis se ubican inmediatamente en el marco general de su explicación por mecanismos de equilibración, respondiendo al modelo de la psicogénesis: "En el curso de la historia del pensamiento científico los progresos logrados de una etapa a la siguiente no se suceden, salvo excepciones, de cualquier manera, sino que pueden ser seriados como en el curso de la psicogénesis, bajo la forma de "estadios" secuenciales". ${ }^{25}$ Para disipar dudas, los autores concluyen su obra

además de no esclarecer en absoluto el misterio de su naturaleza. Por el contrario, el mecanismo mismo de las regulaciones, con sus composiciones y la dirección retroactiva de los "feedbacks", parece ya preparar las estructuras operatorias con su reversibilidad." Piaget, J. y García, R., "Psicogénesis e historia de la ciencia", Méjico, Siglo XXI, 1992, p. 21

${ }^{23}$ Piaget y García, Psicogénesis e historia de la ciencia", op.cit., p. 14 y 15.

${ }^{24}$ Ibid., p. 31

${ }^{25}$ Ibid., p. 33 
recordando que "la ambición permanente de la epistemología genética ha sido mostrar que el desarrollo espontáneo de los conocimientos extrae su fuente de las organizaciones biológicas para llegar en sus etapas más avanzadas a la construcción de las estructuras lógico-matemáticas. Nosotros esperamos que esta obra, al mostrar el papel de la psicogénesis y sus convergencias notables con la historia del pensamiento científico, contribuirá a reforzar tal programa, aún cuando no le hayamos prestado atención al estrecho parentesco que uno de nosotros ha encontrado al efectuar comparaciones posibles entre los mecanismo biológicos y cognoscitivos. A este respecto tenemos que mencionar los trabajos recientes de Prigogine sobre las estructuras disipativas que parecen mostrar la posibilidad de ir aún más lejos y que la serie 'organismo-comportamiento-psicogénesis sensorio-motrizpsicogénesis conceptual', podría ser completada en su parte inferior vinculando las estructuras biológicas (y por consiguiente las cognoscitivas a ciertas formas de equilibrio dinámico que tienen relación con la física (y cuyo estudio ha sido provocado precisamente por la necesidad de vincular entre sí estas dos disciplinas) ". ${ }^{26}$ Es notable, que en una concepción del conocimiento humano que encuentra sus fundamentos explicativos en las organizaciones corporales, no se lleve hasta sus últimas consecuencias lo que significa para el hombre ser corporal.

Aunque Piaget trata de evitar un reduccionismo biologista, en cuanto señala expresamente que las regulaciones diferenciadas de las operaciones superiores no se limitan a los niveles materiales-fisiológicos, la perspectiva biológico-científica es muy fuerte y se ordena a buscar funciones y mecanismos comunes. Las estructuras cognoscitivas en función de la autorregulación son efi-

${ }^{26}$ Ibid., p. 251 
caces en la medida en que la operatividad logra los niveles adecuados de abstracción y generalización. La adaptación cognoscitiva "no es más que un caso particular de las adaptaciones del organismo al medio. En ambos casos, el criterio es el éxito, y a se trate de supervivencia o de comprensión". ${ }^{27}$ La diferencia entre la adaptación intelectual y la adaptación orgánica es que las formas del pensamiento, al aplicarse a distancias crecientes en el espacio y en el tiempo, culminan en la constitución de un "medium" más amplio y más estable. Esta diferenciación y superación en estructuras más refinadas, obtiene sin embargo sus cuadros funcionales de la organización viviente, y desde allí se determina su dirección. La "vección" del proceso se orienta al instrumento más generalizable y con mayor grado de formalización. "Es en la medida en que la inteligencia humana encuentra en las estructuras lógico-matemáticas un instrumento de integración cada vez más independiente de la experiencia como se realiza precisamente la conquista más amplia y más profunda del medio experimentado...Esta depuración de la forma culmina en el terreno cognoscitivo en logros constantemente buscados, valga la expresión, en el campo orgánico, pero nunca alcanzados plenamente". ${ }^{28}$

Piaget centra así el problema en una explicación de la "competencia" cognoscitiva, entendida desde instancias generalizables, más que en la realización histórica del proceso. Por esto su tratamiento de la corporalidad como raíz de la operatividad cognoscitiva superior, no abandona la perspectiva de la "legalidad en tercera persona", ni contempla lo que significa estar "situado" para un sujeto corporal.

${ }^{27}$ Piaget, J., Biología y conocimiento, op.cit., p. 166 ${ }^{28}$ Ibid., op.cit., p. 327-328 
Es preciso entender la facticidad en su justa medida. El cuerpo no sólo me compromete con el mundo de los "organismos estables", sino que define mis coordenadas espaciales y temporales comprometiéndome con la historia. Desde su corporalidad el sujeto se abre a un horizonte significativo que no se limita a la consecución de estructuras generales adaptativas.

El cuerpo es lo que concreta o determina al yo en su facticidad, lo sitúa y particulariza; lo restringe a su finitud y contingencia histórica, a la vez que es instrumento comunicativo y posibilitante de la construcción intersubjetiva del significado del mundo de la cultura en su compleja variabilidad y relatividad. Es lo que inserta al sujeto en una historia de sentido que se despliega teleológicamente. Los análisis piagetianos desdibujan el peso de la corporalidad como la instancia fundamental de la facticidad e historicidad, y por tanto las nociones de espacio y tiempo son despojadas de todo el espesor semántico que tienen en la idea de "horizonte". Si al marco espacio temporal que codetermina el sentido de toda experiencia, lo concebimos además como un horizonte que se despliega según una teleología que se define según proyectos humanos, las motivaciones cobran un significado diferente. El "yo puedo" o "yo quiero", no se interpretan desde mecanismo adaptativos autorregulativos, tendiendo a metas que posibilitan una adaptación más eficaz y equilibrada, sino desde una intencionalidad que se proyecta.

Pero para ello es necesario trascender el nivel de un sujeto epistémico de raíces biológicas que encuentra en el conocimiento el órgano adecuado de adaptación, para entender el cuerpo como órgano de una vida personal. El sujeto entendido en su integridad es miembro de un proyecto compartido (Mitgleid der sozialen Welt $)^{29}$, de una comunidad histórico-social. Desde esta perspecti-

${ }^{29}$ Husserl, E., Ideas II, p. 175 
va, el cuerpo es más que un organismo psico-físico; es -para decirlo con las palabras de Paci- "órgano del espíritu objetivo, de las formas de progresiva realización y de la lucha por la realización del telos en la historia." 30

Si admitimos además que este Telos tiene un carácter normativo, es decir, implica una exigencia de realización, no podemos dejar de señalar no sólo la pobreza de la idea de "adaptación" como rectora de un proceso humano, sino también sus riesgos cuando se aplica al orden social desvinculado de toda dimensión ética.

${ }^{30}$ Paci, E., op.cit., p. 137 